\title{
Nutritional factors and bone health status of retired lead workers in the Republic of Korea
}

\author{
Hee-Seon Kim ${ }^{1}$, Jung Eun Youn ${ }^{1}$, Cheong-Min Sohn ${ }^{2}$, Nam Soo Kim ${ }^{3}$, Andrew Todds ${ }^{4}$, Sung-Soo Lee ${ }^{3}$ \\ and Byung-Kook Lee ${ }^{3}$ \\ ${ }^{1}$ Department of Food Science and Nutrition, Soonchunhyang University, 646 Eupnae-ri, Shinchang-myun, Asan, \\ Choongnam, 336-745, Republic of Korea, ${ }^{2}$ Major in Food and Nutrition, Wonkwang University, 344-2 Sinyong-Dong, \\ Iksan, Jeonbuk, 570-749, Republic of Korea, ${ }^{3}$ Institute of Environmental and Occupational Medicine, Soonchunhyang \\ University, 646 Eupnae-ri, Shinchang-myun, Asan, Choongnam, 336-745, Republic of Korea and ${ }^{4}$ Department of \\ Community and Preventive Medicine, Mt. Sinai School of Medicine, New York, USA
}

The objective of this study was to investigate the nutritional and health status of retired industrial workers. Two-hundred-and-twenty-eight (172 lead and 56 office) workers were recruited from March 2006 to October 2006. Information on age, smoking and drinking status were collected using questionnaire and the nutrient intake of workers was assessed by an average of the 2-d $24 \mathrm{~h}$ recall method. Biochemical indexes including blood lead level $(\mathrm{PbB})$, indexes for Fe status, serum calcium $(\mathrm{Ca})$ and serum lipid profiles were analysed from fasting venous blood or serum. Broadband ultrasound attenuation (BUA) was used as a surrogate of bone mineral density and measured at the left calcaneous bone area. The bone lead contents were also analysed using XRF from the right tibia. Results showed that more drinkers and smokers were reported among retired lead workers than office workers $(P<0.01)$. Tibia lead contents, serum lead, alkaline phosphatase and parathyroid hormone levels of retired lead workers were significantly higher $(P<0.01)$ than that of retired office workers. However, HDL cholesterol and dietary animal calcium intake levels were significantly higher in the control group $(P<0.05)$. Factors affecting BUA most significantly were age, plant fat, BMI, sex, HDL-C, tibia lead. This study confirms that lead workers need extra care to maintain their health and nutritional management. Tailored nutrition education for workers at specific working fields needs to be more focused for the improvement of health status of industrial workers.

This study was supported by Korea Research Foundation Funds (KRP-2004-02-E00048). 